\title{
Versatile attosecond beamline in a two-foci configuration for simultaneous time-resolved measurements
}

Cite as: Rev. Sci. Instrum. 85, 013113 (2014); https://doi.org/10.1063/1.4862656

Submitted: 09 December 2013 . Accepted: 07 January 2014 . Published Online: 24 January 2014

R. Locher, M. Lucchini, J. Herrmann, M. Sabbar, M. Weger, A. Ludwig, L. Castiglioni, M. Greif, M. Hengsberger, L. Gallmann, and U. Keller
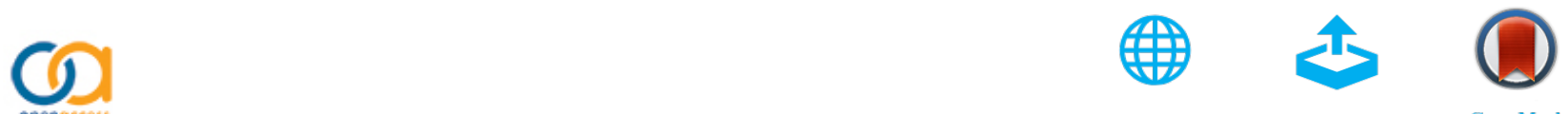

View Online

Export Citation

\section{ARTICLES YOU MAY BE INTERESTED IN}

Attosecond beamline with actively stabilized and spatially separated beam paths

Review of Scientific Instruments 86, 123106 (2015); https://doi.org/10.1063/1.4937623

Combining attosecond XUV pulses with coincidence spectroscopy

Review of Scientific Instruments 85, 103113 (2014); https://doi.org/10.1063/1.4898017

Flexible attosecond beamline for high harmonic spectroscopy and XUV/near-IR pump probe experiments requiring long acquisition times

Review of Scientific Instruments 86, 033108 (2015); https://doi.org/10.1063/1.4914464

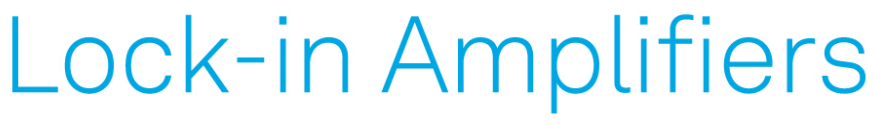
... and more, from DC to $600 \mathrm{MHz}$

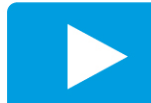

Watch 


\title{
Versatile attosecond beamline in a two-foci configuration for simultaneous time-resolved measurements
}

\author{
R. Locher, ${ }^{1}$ M. Lucchini, ${ }^{1}$,a) J. Herrmann, ${ }^{1}$ M. Sabbar, ${ }^{1}$ M. Weger, ${ }^{1}$ A. Ludwig, ${ }^{1}$ \\ L. Castiglioni, ${ }^{2}$ M. Greif, ${ }^{2}$ M. Hengsberger, ${ }^{2}$ L. Gallmann, ${ }^{1}$ and U. Keller ${ }^{1}$ \\ ${ }^{1}$ Department of Physics, ETH Zurich, CH-8093 Zürich, Switzerland \\ ${ }^{2}$ Institute of Physics, University of Zurich, CH-8057 Zürich, Switzerland
}

(Received 9 December 2013; accepted 7 January 2014; published online 24 January 2014)

\begin{abstract}
We present our attoline which is a versatile attosecond beamline at the Ultrafast Laser Physics Group at ETH Zurich for attosecond spectroscopy in a variety of targets. High-harmonic generation (HHG) in noble gases with an infrared (IR) driving field is employed to generate pulses in the extreme ultraviolet (XUV) spectral regime for XUV-IR cross-correlation measurements. The IR pulse driving the HHG and the pulse involved in the measurements are used in a non-collinear set-up that gives independent access to the different beams. Single attosecond pulses are generated with the polarization gating technique and temporally characterized with attosecond streaking. This attoline contains two target chambers that can be operated simultaneously. A toroidal mirror relay-images the focus from the first chamber into the second one. In the first interaction region a dedicated double-target allows for a simple change between photoelectron/photoion measurements with a time-of-flight spectrometer and transient absorption experiments. Any end station can occupy the second interaction chamber. A surface analysis chamber containing a hemispherical electron analyzer was employed to demonstrate successful operation. Simultaneous RABBITT measurements in two argon jets were recorded for this purpose. () 2014 Author(s). All article content, except where otherwise noted, is licensed under a Creative Commons Attribution 3.0 Unported License. [http://dx.doi.org/10.1063/1.4862656]
\end{abstract}

\section{INTRODUCTION}

The discovery of high-harmonic generation ${ }^{1,2}$ (HHG) has provided the scientific community with a valuable tool to access the extreme ultraviolet (XUV) spectral region with tabletop sources. Since the temporal characteristics of the XUV radiation bursts generated with HHG naturally lie in the sub-femtosecond domain, this new technology has paved the way for the development of attosecond science. ${ }^{3-6}$ While in femtochemistry ${ }^{7}$ optical femtosecond pulses are used to study nuclear motion in chemical reactions, attosecond science can access the much faster electron dynamics directly in the time domain. Indeed, a comb of odd-order harmonics as generated by HHG corresponds to an attosecond pulse train (APT) in the time domain. ${ }^{8,9}$ Isolating single attosecond pulses (SAPs) from the APT facilitates the study of electron dynamics further and many different gating schemes have been proposed to this end. ${ }^{10}$

Independent of the applied gating mechanism the available flux in SAPs is still rather small and only very few true attosecond pump-attosecond probe experiments ${ }^{11-13}$ have been realized to date. Because of the limited average photon flux in the XUV regime, most attosecond measurements have been based on cross-correlation measurements between the XUV pulse and its driving pulse in the infrared (IR). Hereby, the synchronized IR field is employed as a temporal reference against which the electron/ion dynamics is measured. In the past, many measurement schemes employing this

a) Author to whom correspondence should be addressed. Electronic mail: mlucchini@phys.ethz.ch idea have been suggested and experimentally implemented. These schemes either probe the ultrafast dynamics by detecting charged particles ${ }^{9,14}$ created after the absorption of the pump pulse or by recording the change in absorption of the XUV radiation..$^{15}$ More recently attosecond measurements have been also obtained with the attoclock technique ${ }^{16,17}$ to resolve the electron tunneling delay time ${ }^{17,18}$ and the timing of double ionization ${ }^{19}$ in noble gases. Furthermore, interferometric methods have been used to resolve the ionization delay between two states in noble gases. ${ }^{20}$

In addition to the increasing number of attosecond measurement techniques demonstrated on noble gas targets, a growing number of more complex physical systems have been investigated as well. The first attosecond measurements were conducted in rare gas atoms to temporally characterize the XUV pulses..$^{9,21}$ Soon attosecond spectroscopy was extended to study molecules ${ }^{22}$ and condensed-matter systems. ${ }^{23}$ Here, we describe the attoline at ETH Zurich that can support many different measurement techniques with targets ranging from noble gases to thin films and solid-state surfaces. This beamline has the necessary flexibility to quickly change between the different targets during operation. Moreover, simultaneous measurements on two targets are possible. This unique feature can be used to provide a reference for a more complex target with respect to a better-understood noble gas sample.

In 2010, Fieß et al. ${ }^{24}$ reported on their beamline for time resolved pump-probe experiments in gas phase with attosecond resolution. A year later Magerl et al. ${ }^{25}$ presented a beamline for attosecond photoelectron spectroscopy of solids and surfaces. Their apparatus allows studying dynamics in a large variety of surfaces and interfaces and is equipped with 
state-of-the-art preparation and characterization tools. However, its application is limited to surfaces. In both beamlines, SAPs are generated by spectral filtering of the harmonic radiation around the cutoff. ${ }^{26}$ While this technique yields attosecond pulses at high photon energies, its photon flux is inherently low. Recently, Frank et al. ${ }^{27}$ described a beamline, suitable for single attosecond pulse generation, based on the same spectral filtering technique. All these setups are equipped with a single interaction region in which pumpprobe measurements can be performed.

In contrast our attoline is a versatile attosecond beamline comprising two separate interaction regions. In the first interaction region it is possible to perform pump-probe measurements on a gas target with photoelectron or photoion detection as well as with transient absorption spectroscopy. The second interaction region is an image of the first one and can be occupied by any end station, offering full flexibility for diverse collaborations. In a first experiment a surface physics end station was installed to prove that the demanding background pressure requirements for photoemission studies from surfaces can be fulfilled. In this review, we will show that the adopted refocusing scheme preserves the spatio-temporal properties of both the XUV and the IR pulses enabling simultaneous measurements in the two interaction regions. In contrast to the previously mentioned beamlines, the XUV-generating pulse and the IR pulse for cross-correlation measurements are separated prior to the high-harmonic generation. This offers full flexibility as the parameters of the two beams like pulse energy, temporal duration, and polarization can be adjusted independently. This flexibility is further supported by the fact that only metallic mirrors and in particular no XUV bandwidth limiting components are used throughout our beamline. This overall design allows us to apply the polarization gating (PG) technique ${ }^{28}$ for SAP generation in a straightforward fashion. The temporal characterization of such pulses by the attosecond streaking technique ${ }^{14}$ will be shown.

This paper is divided into four main sections. Section II presents a detailed description of the experimental setup of our attoline. In Sec. III, experimental results obtained with our attoline are reported. Section IV contains the conclusions.

\section{EXPERIMENTAL SETUP}

High-order harmonics $(\mathrm{HH})$ are generated by ultrashort IR pulses from a Ti:sapphire laser based chirped-pulse amplifier system (Femtopower V CEP, Femtolasers). The $1 \mathrm{kHz}$ laser system comprises two consecutive amplifier stages delivering separate output beams that can be operated simultaneously. Active stabilization of the carrier-envelope-phase ${ }^{29}$ (CEP) is possible for one beam at a time. While the output after the first amplification stage is characterized by a pulse duration of $25 \mathrm{fs}$ and a pulse energy up to $1.3 \mathrm{~mJ}$, the second stage amplifies the pulses further up to an energy of $10 \mathrm{~mJ}$ keeping the time duration of the pulses almost unchanged ( $\sim 30 \mathrm{fs}$ ). Each output beam propagates through an evacuated tube to the coupling chamber (see Fig. 1) of the beamline where the $\mathrm{HH}$ are generated.

The experimental setup of our attosecond beamline (termed "attoline") is presented in Fig. 1. Turbomolecular pumps of various sizes maintain our beamline under vacuum. For the sake of clarity the attoline is divided into four parts (delimited by the frames in the figure) and discussed separately in Subsections II A-II D.

\section{A. Coupling and generation chambers}

The IR pulses are coupled to the attoline via a dedicated vacuum chamber (i.e., the coupling chamber, Figs. 1 and 2). As previously mentioned, the two outputs of the laser system are independently sent through two evacuated tubes (T1 and T2). To avoid long-term beam-pointing instabilities the last mirror before each tube is actively stabilized with a home-built beam-stabilization system. The coupling chamber is designed such that switching between the two available driving pulses does not entail a major realignment of the

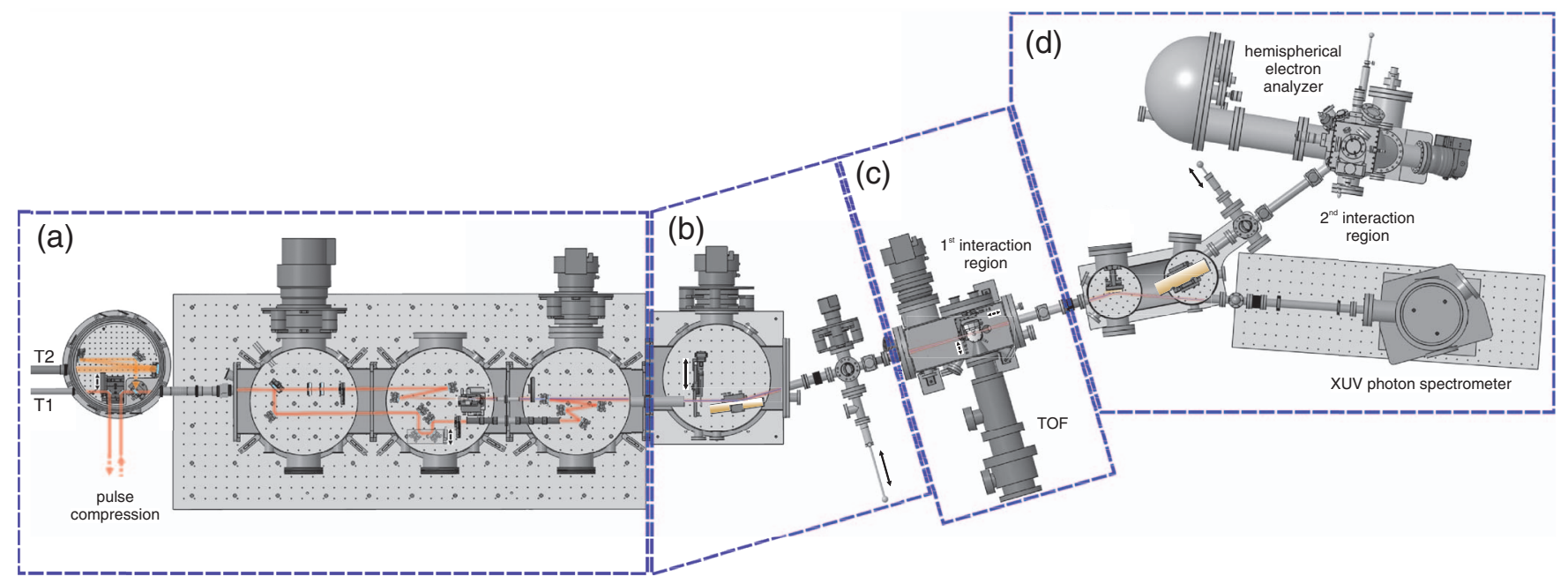

FIG. 1. Experimental setup consisting of four main blocks (delimited by the dashed frames: (a) laser beam coupling and XUV generation chambers, (b) diagnostics and focusing chamber, (c) first interaction chamber with a time-of-flight (TOF) spectrometer, and (d) second refocusing and interaction chambers together with the photon spectrometer. The hemispherical electron analyzer placed in the second interaction region is not a permanent component of the attoline, but represents an example for an exchangeable end station (see Sec. III B). 


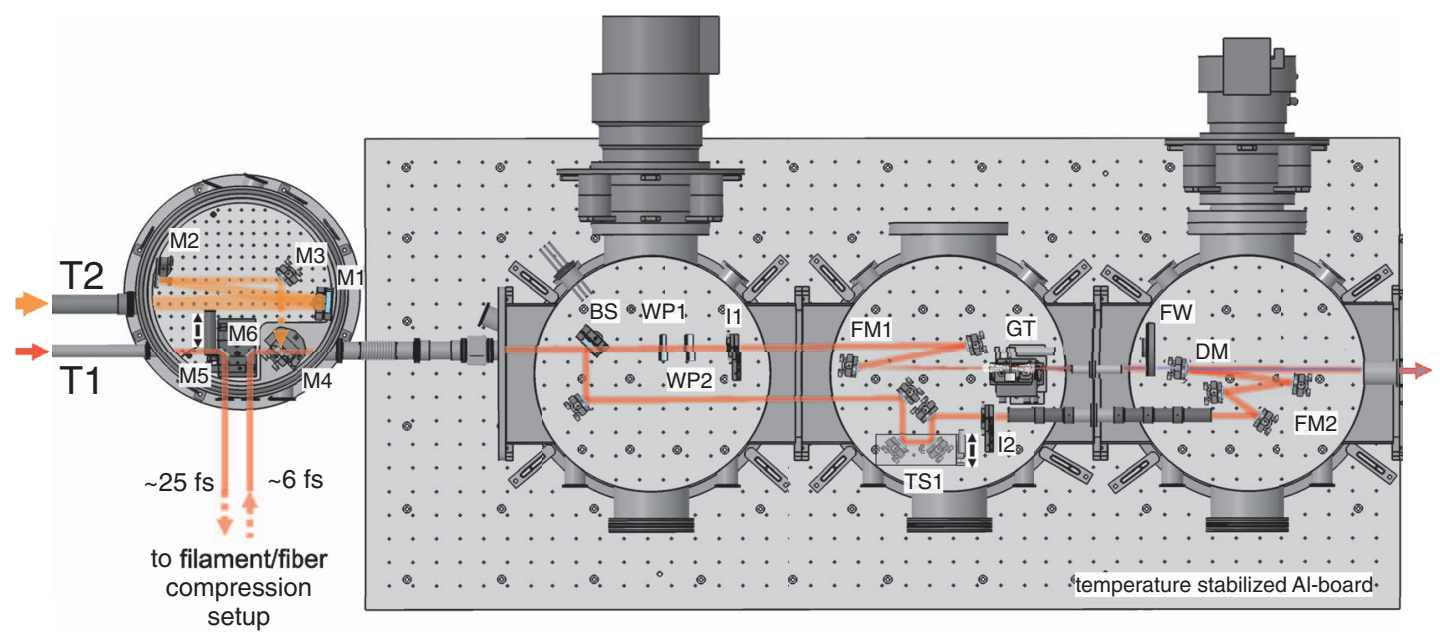

FIG. 2. Coupling and generation chambers: In the first chamber the two outputs from the laser system are coupled into the attoline. The three adjacent identical chambers form the pump-probe interferometer. The IR beam is split into two parts by a beam splitter (BS) located in the first chamber. The more intense part passes the beam splitter and is focused into the gas target (GT) to generate high-order harmonics. The weaker part is delayed and subsequently recombined with the XUV beam with a drilled mirror (DM) which contains a small center hole for the XUV beam and still reflects most of the larger IR beam. For improved stability the entire interferometer is placed onto a temperature-stabilized aluminum board on top of a standard commercial laser table.

beamline (see Fig. 2). If the mirrors are set as in Fig. 2, the $10-\mathrm{mJ}$ output of the laser system is coupled into the attoline. In order to minimize the detrimental effect of nonlinearities in air and at the transmission through the entrance window of the tube, the beam size of the 10-mJ pulses is chosen considerably larger than for the low-energy output.

The coupling chamber contains a telescope composed of mirrors M1 and M2 to adjust the beam size to smaller optics. The beam is subsequently steered into the attoline by the mirrors M3 and M4. The last mirror is vertically movable. By removing M4 from the beam path, the $10-\mathrm{mJ}$ output is blocked, but the $1.3-\mathrm{mJ}$ beam coming from $\mathrm{T} 1$ is coupled into the attoline. Two additional mirrors, M5 and M6, are mounted on a translation stage and can be used to extract the beam from the coupling chamber in order to compress the pulses.

In the two experiments explained in Secs. III A and III B, the second scheme was adopted and $1.3-\mathrm{mJ}$ pulses were sent through two filament cells filled with argon for spectral broadening. ${ }^{30,31}$ The residual group-delay dispersion was compensated with broadband chirped mirrors. Pulses with energies of $\sim 350 \mu \mathrm{J}$ and a time duration down to $\sim 5.5$ fs were achieved. A second compression setup based on a 1-m long hollow-core fiber with a diameter of $300 \mu \mathrm{m}$ is available. ${ }^{32}$ While the filament-based setup is more robust to any instabilities of the beam pointing, the hollow-core fiber enables a more efficient coupling and delivers sub 6-fs pulses with an energy of $\sim 600 \mu \mathrm{J}$. In both cases, the compressed pulses are coupled back into the attoline with the mirror M6.

Next to the coupling chamber there are three identical cylindrical vacuum chambers (inner diameter of $0.5 \mathrm{~m}$ ) that contain the interferometer setup for preparing the XUV/IR pump-probe measurements. The top lids are made of acrylic glass (50-mm thick) and make visual inspection under operation conditions possible. Rectangular flanges interconnect the chambers in order to increase the intersection area for flexible beam routing. Custom gates, specifically designed to optimize the differential pumping according with the beam path geometry, can be easily inserted in the rectangular flanges. Sufficient mechanical stability of the interferometer is assured without the need for active stabilization by attaching the chambers to a $60-\mathrm{mm}$ thick single-piece temperature stabilized aluminum board $\left(2.2 \times 1.0 \mathrm{~m}^{2}\right)$. Possible vibrations due to the turbomolecular pumps are strongly attenuated with dampers. This board is equipped with a $25 \mathrm{~mm} \times 25 \mathrm{~mm}$ M6 thread grid to allow for free configuration of the optics in the three chambers. O-rings are employed for the sealing with respect to this baseplate and the acrylic lid.

In the first chamber, the incoming beam is divided by a beam splitter (BS in Fig. 2), where $80 \%$ of the pulse is transmitted and $20 \%$ reflected. The transmitted beam, adopted for the XUV-light generation, propagates towards the second chamber where it is focused by a concave mirror (FM1, ROC $=-750 \mathrm{~mm}$ ) into a gas target $(\mathrm{GT})$. The gas target consists of a metal capillary that is closed on one end and possesses two traversal holes for the beam passage. For minimal gas load the holes are sealed with a Teflon tape that is perforated by the laser beam in situ. Mounting the target on a motorized $x y z$-stage and sending the beam through an adjustable iris prior to focusing (I1), allows us to optimize the phase matching of the HHG process in real time and hence tailor the XUV signal according to the experimental requirements. A wheel containing different metallic filters (FW) is used to eliminate the residual IR radiation from the harmonic signal. The beam reflected at the beam splitter (BS) is used as the IR reference for pump-probe experiments. It follows a delayed path and is subsequently recombined with the XUV light by means of a drilled mirror (DM) placed in the third chamber. A motorized broadband half-wave plate (not shown) can be used to change the polarization direction of the beam under operating conditions. The last mirror (FM2) of this arm of the interferometer is convex $(\mathrm{ROC}=500 \mathrm{~mm})$ in order to match the divergence between the XUV and the IR beam. The delay between the two arms of the interferometer is controlled with a stack of two different translation stages (TS1). A first stage 
(ECS3080/NUM/HV, attocube) enables to span the delay over a 300-ps range with a resolution of $1 \mathrm{fs}$. For a finer temporal resolution a piezoelectric actuator (PX100CAP, Piezosystem Jena) in closed-loop operation is used. A voltage increment of $10 \mathrm{mV}$ on this piezo actuator corresponds to an increase in delay of 21 as. Finally, mirrors DM and FM2 are motorized and enable for the spatial overlap between the two beams to be optimized. Note that the free space left in the first chamber can host any setup suitable to control and tailor the optical properties of the two beams. This makes the attoline extremely flexible. Indeed, this feature is exploited to install a polarization-gating setup (WP1 and WP2 in Fig. 2) and generate single attosecond pulses ${ }^{33}$ as described in Sec. III A of this work.

\section{B. Diagnostics and focusing}

The next chamber (Fig. 3) houses the toroidal mirror (TORO1, Carl Zeiss Laser Optics GmbH) for focusing the IR and XUV beams into the first target region. It consists of a gold-coated (layer thickness $40 \mathrm{~nm}$ ) Zerodur substrate with toroidal radii $R_{\text {meridial }}=8529.6 \mathrm{~mm}$ and $R_{\text {sagittal }}=165.2 \mathrm{~mm}$. At our incidence angle of $82^{\circ}$ this corresponds to a symmetrical imaging with an arm length of $1187 \mathrm{~mm}$. As the two beams enter the chamber collinearly with matching divergence, both beams are focused to the same spot by the toroidal mirror. Indeed, the toroidal mirror creates a one-to-one image of the HHG target (GT). Since no XUV bandwidth limiting components (e.g., no back-reflecting dielectric XUV mirror) are used in our beamline, switching between different photon energies and different temporal characteristics (APT, SAP) is straightforward and renders our source highly flexible.

Moreover, the chamber contains two instruments for diagnostics of the XUV radiation: a high-resolution beam imaging system sensitive in the XUV (Model BIS-1135 with BIS1-PH probe head with BIS-4135 remote system, Colutron) and a calibrated photodiode (windowless far UV photodi-

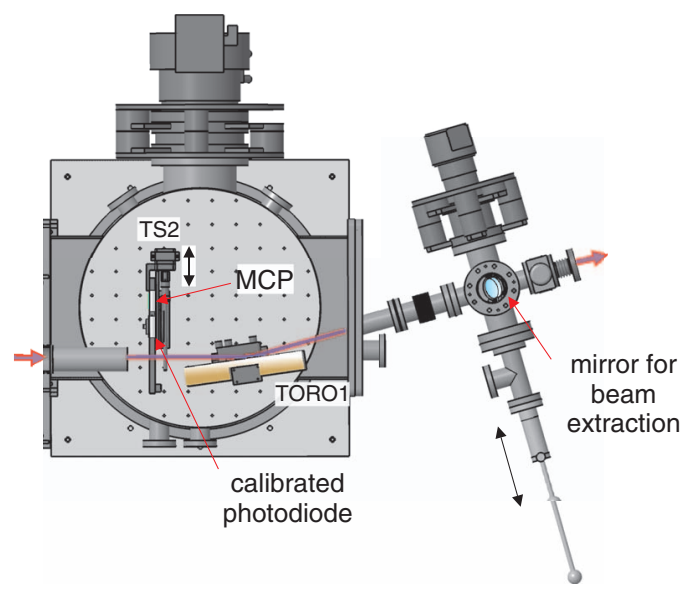

FIG. 3. Chamber with focusing toroidal mirror and XUV beam diagnostics. A MCP-based XUV beam profiler and a calibrated XUV photodiode are mounted on a translation stage (TS2) and can be moved into the beam path with a stepper motor. The smaller cross chamber hosts a mirror mounted on a push-pull manipulator that is used to extract and inspect the pump and probe beams. ode, NIST). Both devices are mounted on a motorized translation stage (TS2) and can be inserted into the beam path when needed. The beam profiler consists of a micro channel plate (MCP) stacked onto a phosphor screen and can be used to characterize the spatial properties of the XUV beam and to guard against detrimental effects of the homogeneity of the metal filters on the beam profile. The calibrated photodiode comprises a cylindrical stainless steel anode and a photo cathode, which is formed by high-purity aluminum on a polished quartz substrate. Accounting for the quantum efficiency the current from this photodiode yields the absolute photon flux. This current is accurately measured by an electrometer (Keithley).

Finally, a silver mirror can be inserted into the converging beams with a push-pull manipulator to extract the beams from the beamline and adjust the temporal and spatial overlap. Obviously, the XUV beam is hereby represented by the residual driving IR pulse that co-propagates collinearly with the high-order harmonics after rotating the metal filter wheel to an empty spot. The temporal overlap is assessed with spectral interferometry.

\section{First interaction chamber}

The two beams are focused into a dedicated chamber for photoelectron/photoion spectroscopy and transient absorption experiments primarily in gas phase targets. An internal $\mu$ metal shielding reduces the magnetic flux down to $\approx 4 \mu \mathrm{T}$ for photoelectron measurements. For a further reduction of the magnetic flux a second shielding (closer to the actual interaction area) can be inserted. In the effort to make the transition between attosecond transient absorption measurements ${ }^{34-36}$ and photoelectron/photoion spectroscopy as straightforward and convenient as possible, we designed a movable double target (Fig. 4). It is composed of two orthogonal targets. The target for transient absorption has a small cylindrical cell 1.5-mm long and with a diameter of $300 \mu \mathrm{m}$, placed on top of the pulsed piezo-valve working at $1 \mathrm{kHz}$ repetition rate and synchronized with the laser system. Such a cell is suitable for providing high particle densities during interaction with the

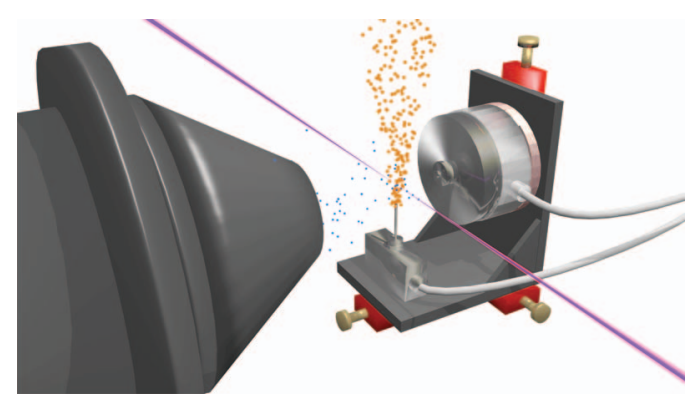

FIG. 4. Double target installed in the first interaction chamber. The target for photoelectron/photoion spectroscopy (center) consists of a stainless steel needle connected to a gas reservoir and provides a continuous gas flow. The TOF spectrometer (left) detects the charged particles. For transient absorption spectroscopy a pulsed gas target (right) is used, which is synchronized to the laser repetition rate. The pulsed operation in combination with the "T-shaped" target tip (diameter $300 \mu \mathrm{m}$, length $1.5 \mathrm{~mm}$ ) provides a high gas density for absorption experiments. Both gas targets are mounted on a $x y z$-translation stage and can be moved relative to the beam. 
laser pulses while keeping the total gas load low. ${ }^{37}$ The tip of the second target is a stainless-steel needle with internal diameter of $240 \mu \mathrm{m}$ through which the gas is injected into the chamber. In contrast to the transient absorption target, the open geometry of this second target allows for efficient collection of charged fragments created by the pump pulse. In particular, the needle target has been adopted in combination with a time-of-flight (TOF) spectrometer (electron time-offlight ETF11, Stefan Kaesdorf) to characterize the temporal properties of the XUV radiation by performing RABBITT ${ }^{9,38}$ and FROG-CRAB measurements ${ }^{21}$ (see Sec. III A). The double target is mounted on a xyz-stage to quickly switch between the two different targets and achieve optimal spatial overlap with the laser pulses.

For photoelectron/photoion measurements a low background pressure is essential. With the high gas density in the HHG site several differential-pumping stages were implemented to prevent the generation gas from entering the interaction chamber. For this purpose metal plates with small apertures for the beams were inserted into the rectangular flanges between the chambers described in Sec. II A. In this way pressures as low as $5 \times 10^{-8}$ mbar were achieved in the interaction chamber with pressures up to $3 \times 10^{-3}$ mbar in the chamber of the HHG.

\section{Refocusing and second interaction chamber}

After the interaction chamber the two beams enter a second refocusing chamber (Fig. 5) with two possible beam paths. The spherical mirror (SM) astigmatically (incidence angle of $80^{\circ}, \mathrm{ROC}=8 \mathrm{~m}$ ) focuses the XUV beam along path 1 in Fig. 5 onto the entrance slit (width of $100 \mu \mathrm{m}$ ) of the toroidal grating flat field spectrometer (McPherson Model 251). The grating has 450 grooves $/ \mathrm{mm}$ and allows for the detection of photon energies up to $124 \mathrm{eV}$. For improved resolution and higher photon energies the grating can be exchanged for one with 1200 groves $/ \mathrm{mm}$. The spectrally dispersed XUV beam is detected with a Peltier-cooled CCD (PIXIS-XO, 400B, Princeton Instruments). As the latter is sensitive to IR radiation, additional metallic filters are

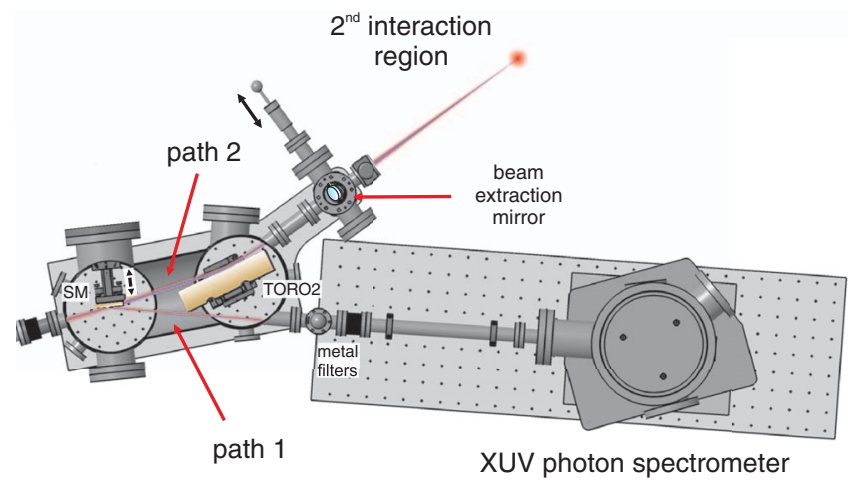

FIG. 5. Second refocusing chamber and the two possible beam paths. When the spherical mirror (SM) is inserted, the beam is astigmatically focused onto the entrance slit of the XUV photon spectrometer (path 1). With the spherical mirror SM retracted the beams co-propagate onto the second toroidal mirror (TORO2) and are focused into the second interaction region (path 2). installed after the spherical mirror to block the probe beam. Note that the spectrometer can be operated during any measurement in the first target chamber.

The spherical mirror is mounted on a motorized translation stage (TS3, ECS3070, attocube) and can be removed (and inserted) from the beam path under operating conditions. Indeed, if the spherical mirror is retracted, the two beams copropagate along path 2 in Fig. 5 towards the second toroidal mirror (TORO2) and are refocused into the second interaction region. More precisely, the toroidal mirror relay-images the first focus into the second interaction region. The toroidal mirror is of the same composition as the first one with $R_{\text {meridial }}$ $=5758.7 \mathrm{~mm}$ and $R_{\text {sagittal }}=173.6 \mathrm{~mm}$. Symmetrical imaging under an incidence angle of $80^{\circ}$ results in an arm length of $1000 \mathrm{~mm}$.

Any other experiment can now be attached at the end path 2 in Fig. 5 of our attoline. This opens the door for a variety of possible experiments. The design of the connection with the end station presented in Sec. III B allowed us to reach pressures well below $10^{-9}$ mbar in the second interaction region. This is suitable for experiments on condensed matter systems, where ultra-high vacuum conditions are required to avoid contamination of the sample. In particular, thanks to the great flexibility of our setup, it is possible to exploit the interaction with a gas target in the first focal point to characterize the temporal characteristics of the pump and probe pulses and subsequently perform pump-probe measurements in the second focal point with a completely different target. Owing to efficient differential pumping of the target gas in the first interaction region, measurements can be conducted simultaneously with the extension presented in Sec. III B. This scheme enables us to understand whether the features measured in the second interaction point are ascribable to the particular characteristics of the pump/probe beams or if they are the peculiar fingerprints of the physical system under investigation.

\section{MEASUREMENTS}

The functionality of the presented attoline is demonstrated with two particular measurements. In Sec. III A the name "attoline" is justified by reporting on the generation and full temporal characterization of SAPs. This substantiates the versatility of our beamline on the source side. In Sec. III B, an XUV-IR cross-correlation measurement in the second interaction region with attosecond pulse trains is presented. A RABBITT $^{9,38}$ trace is recorded in an Ar gas target placed in the second interaction region and shows stable oscillations of the sidebands. This demonstrates the high fidelity of the reimaging with the second toroidal mirror.

\section{A. Attosecond streaking measurement}

We generated single attosecond pulses based on the PG technique. ${ }^{28,33}$ As the HHG process exhibits a high sensitivity to the polarization of the driving field, it is possible to temporarily confine the XUV light generation by properly shaping the polarization of the IR driving pulse. For an ellipticity of the driving field of $\varepsilon_{t h}=0.13$ the efficiency of 
(a)

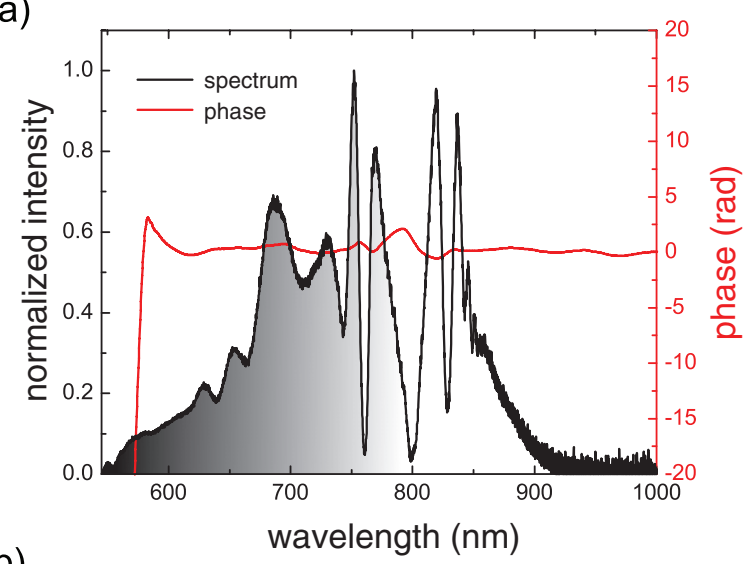

(b)

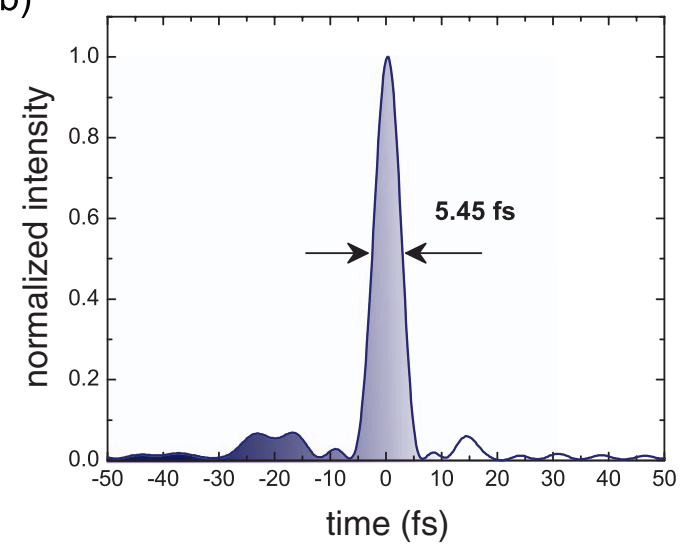

FIG. 6. (a) Intensity (black line) and reconstructed phase (red line) of the spectrum associated to the driving pulses after the filament compression setup. (b) Spectral Phase Interferometry for Direct Electric-field Reconstruction (SPIDER) of the pulse. The reconstruction shows few-cycle pulses with a pulse length of $5.5 \mathrm{fs}$, close to its transform-limit.

the process decreases by $50 \%$ with respect to the case of linear polarization. ${ }^{10}$ In particular, single attosecond pulse selection is achieved by a driving pulse that is linearly polarized in a time window shorter than the temporal separation of consecutive attosecond bursts. Among all the possible setups proposed in literature, ${ }^{4}$ a linear one composed of a retarding quartz plate followed by a zero-order quarter wave plate $^{39}$ (WP1 and WP2 in Fig. 2) was adopted. By a proper choice of the angle between the polarization axis of the driving pulses and the optical axis of the plates, it is possible to obtain an IR pulse that is circularly polarized on the leading and trailing edges and linearly polarized in the center. A sufficiently narrow gate for the isolation of a single attosecond pulse can be obtained either by reducing the pulse duration or by increasing the temporal delay between the two orthogonal polarization states. Large values of $\delta$ will deeply affect the efficiency of the generation process by reducing the amplitude of the electric field. Therefore, driving pulses with a time duration $\tau$ shorter than $7 \mathrm{fs}$ are required for an efficient generation of SAPs with the PG technique. ${ }^{40}$ A filament based compression setup was employed to reduce the time duration of the pulses down to $5.5 \mathrm{fs}$, as described in Sec. II A (see Fig. 6). Besides the short pulse duration, a fixed and appropriate CEP of the driving pulse is required for the PG technique to work. The CEP is adjusted with a pair of fused silica
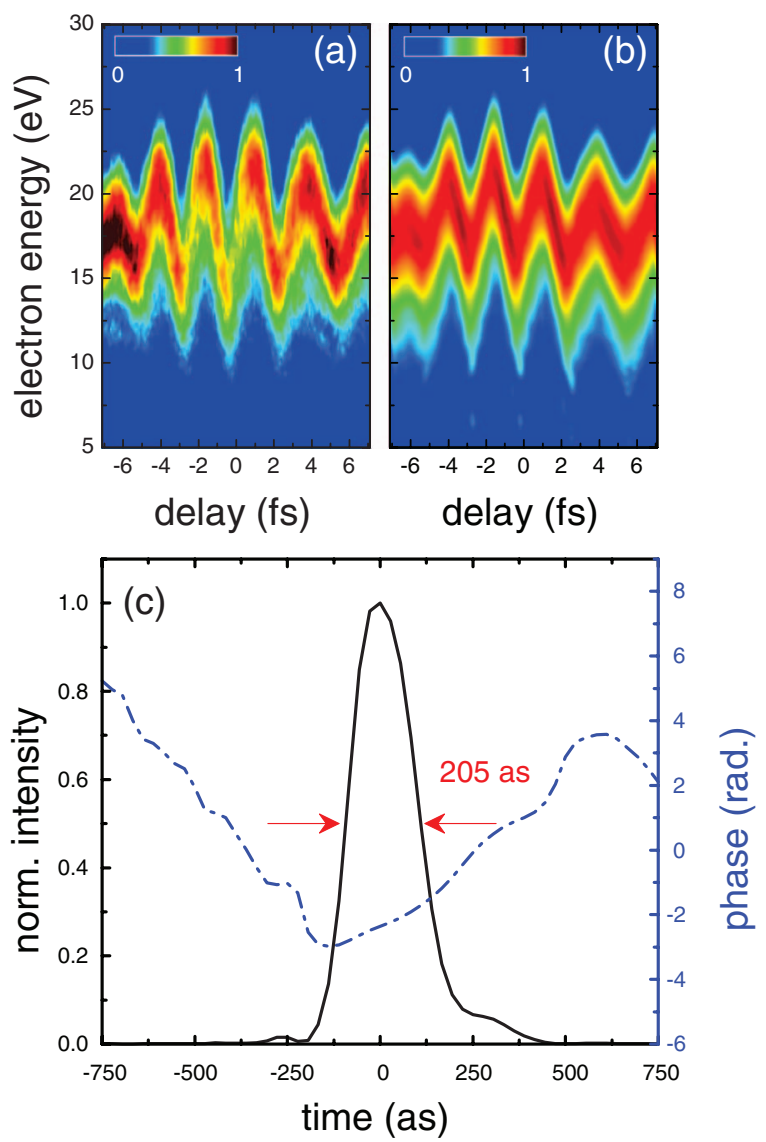

FIG. 7. (a) Experimental spectrogram obtained with a single attosecond pulse generated with the PG technique after a $100-\mathrm{nm}$ thick Al filter. (b) Reconstructed FROG-CRAB trace after $8 \times 10^{5}$ iterations of the retrieval algorithm. (c) Temporal profile, intensity and phase, of the reconstructed pulse. The retrieved pulse shows a time duration of 205 as.

wedges placed before the filament compression setup. Depending on the CEP the PG produces single or multiple attosecond pulses and hence provides a convenient means to alter the temporal structure of our XUV radiation source. We have used this APT control for transient absorption measurements to influence the optical response of helium atoms to XUV radiation using APTs with reducing numbers of attosecond pulses down to the SAP limit. ${ }^{35}$

The attosecond streaking technique ${ }^{14}$ was applied to determine the temporal characteristics of our SAPs. For this purpose argon gas was injected into the first interaction chamber using the needle target. Figure 7(a) shows the obtained experimental spectrogram. The temporal characteristics of the attosecond pulse were retrieved from the experimental spectrogram with an appropriate iterative algorithm. ${ }^{41}$ The retrieved FROG-CRAB trace obtained after $8 \times 10^{5}$ iterations of the algorithm is shown in Fig. 7(b). The error between the retrieved and the experimental traces, evaluated as the root-mean-square error per element of the trace, ${ }^{42}$ is $<2 \times 10^{-3}$. The reconstructed temporal profile displayed in Fig. 7(c) shows a single attosecond pulse with a time duration (FWHM) of $\sim 205$ as (transform limit $\sim 180$ as). It is noteworthy that an aluminum filter of adequate thickness could further decrease the residual chirp. ${ }^{43}$ 


\section{B. Double RABBITT}

As described in Sec. II D, the second toroidal mirror images the XUV and the IR beams from the first focus into the second focus and hence cross-correlation measurements between the two pulses can be performed in the second interaction region like in the first one. In this section, we demonstrate the feasibility of such a measurement. In contrast to the streaking described in Sec. III A, attosecond pulse trains were used for this measurement. Moreover, the IR probe pulse was much weaker. The reduction in probe power was achieved by closing the appropriate iris (I1 in Fig. 2) in the beam path of the probe pulse.

As mentioned above, we extended the attoline with a second target in order to be able to study condensed-matter systems. For this purpose, a surface end station comprising a hemispherical electron analyzer (Phoibos 150 WAL, SPECS $\mathrm{GmbH}$ ) was installed in the second interaction region to measure photoemission spectra from noble metal surface samples. The experimental station is equipped with full standard surface preparation and characterization techniques comprising low-energy electron diffraction (LEED) and x-ray photoelectron spectroscopy (XPS). The sample is mounted on a $2 \pi$ goniometer that allows for electron detection from all possible emission angles from the surface. An entry load lock allows for fast sample exchange. Details of this apparatus will be published elsewhere. In brief, a 2D CCD-type detector is used for electron detection in the hemispherical analyzer. The dimensions of the detector represent the energy dispersion (horizontal axis) and the emission angle (vertical axis) in a range of $\pm 30^{\circ}$ with respect to the analyzer axis and perpendicular to the plane of incidence of the incoming light. The resolution of the electron analyzer was determined to be $<5 \mathrm{meV}$ and $<0.5^{\circ}$ in energy and angle, respectively. The angular resolution in the energy-dispersive direction of the analyzer depends on the entrance slit. The entrance slit width is chosen as a trade-off between electron count rate and angular and energy resolution. The angle between light incidence and analyzer axis is $45^{\circ}$. The interface between the attoline and the surface end station was designed such that simultaneous RABBITT measurements in both interaction regions are possible.

In order to test the temporal and spatial properties of the two beams in the second target, we first performed a simultaneous measurement of two RABBITT traces from argon atoms. For this purpose, a nozzle identical to the one hosted in the first interaction (Sec. II C) region has been installed in the second one.

In a RABBITT measurement, electrons at energies corresponding to even harmonics (so-called sidebands) appear as the result of two-photon two-color transitions involving an XUV and an IR photon. Furthermore, the electron yield at these energies oscillates with the delay between the two pulses due to quantum path interference. . $^{9} 38$

Figure 8 shows simultaneously recorded RABBITT traces in the two target regions. Sidebands clearly appear in both traces, demonstrating that the reimaging results in excellent spatial overlap and shape of the two pulses. Furthermore, the oscillations persist over the total scan, only (a)

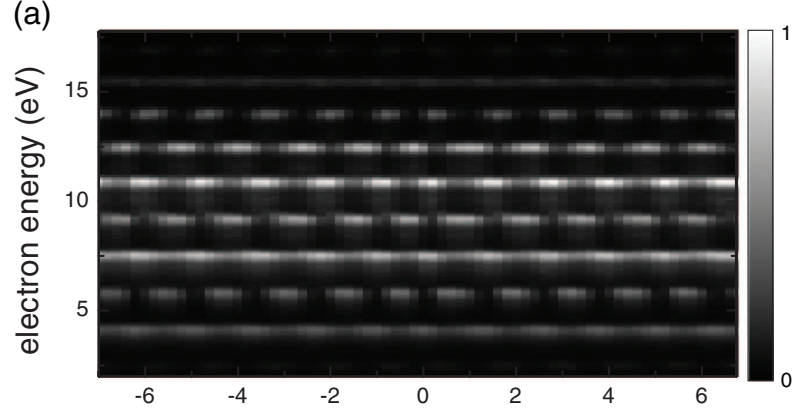

(b)

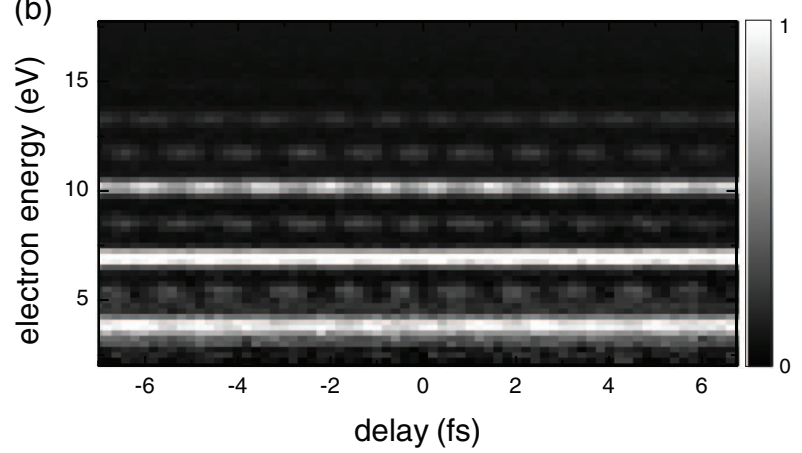

(c)

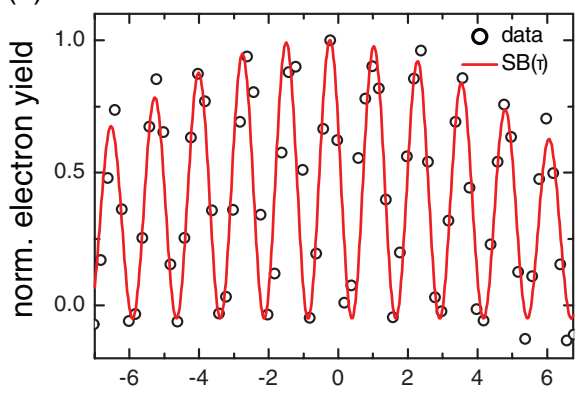

(d)

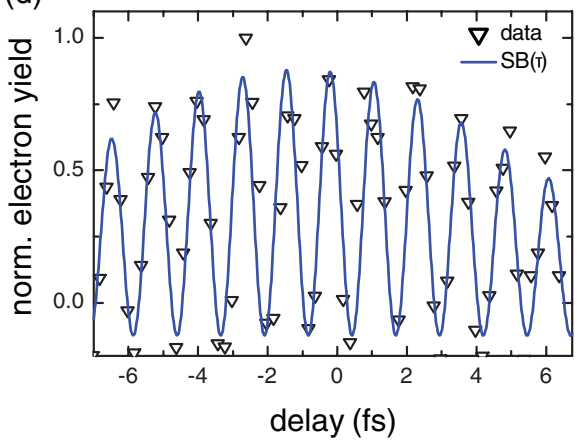

FIG. 8. RABBITT traces recorded simultaneously in argon in the first (a) and second (b) interaction region. For better visibility of the sidebands (SBs) on the second gas target, the background signal due to secondary electrons was subtracted. Sidebands appear in the interaction region at around $5.6 \mathrm{eV}, 9 \mathrm{eV}, 12.3 \mathrm{eV}$, and $15.5 \mathrm{eV}$ corresponding to harmonic orders 14 to 20 . At the same time, the harmonic peaks are depleted. The normalized electron yields integrated over an energy band of $0.5 \mathrm{eV}$ centered around SB 18 are shown in (c) and (d) for the first and second target, respectively. The data are fitted with an oscillatory function $S B(\tau)=A \cdot e^{-\left(\frac{\tau-\tau_{0}}{w}\right)^{2}} \cdot \cos ^{2}\left(\omega_{0} \tau-\varphi\right)$ (where $\tau$ is the delay and $\omega_{0}$ is the carrier angular frequency) for the sake of a better understanding, and clearly demonstrate that the sub-cycle resolution is preserved in the second interaction region. 


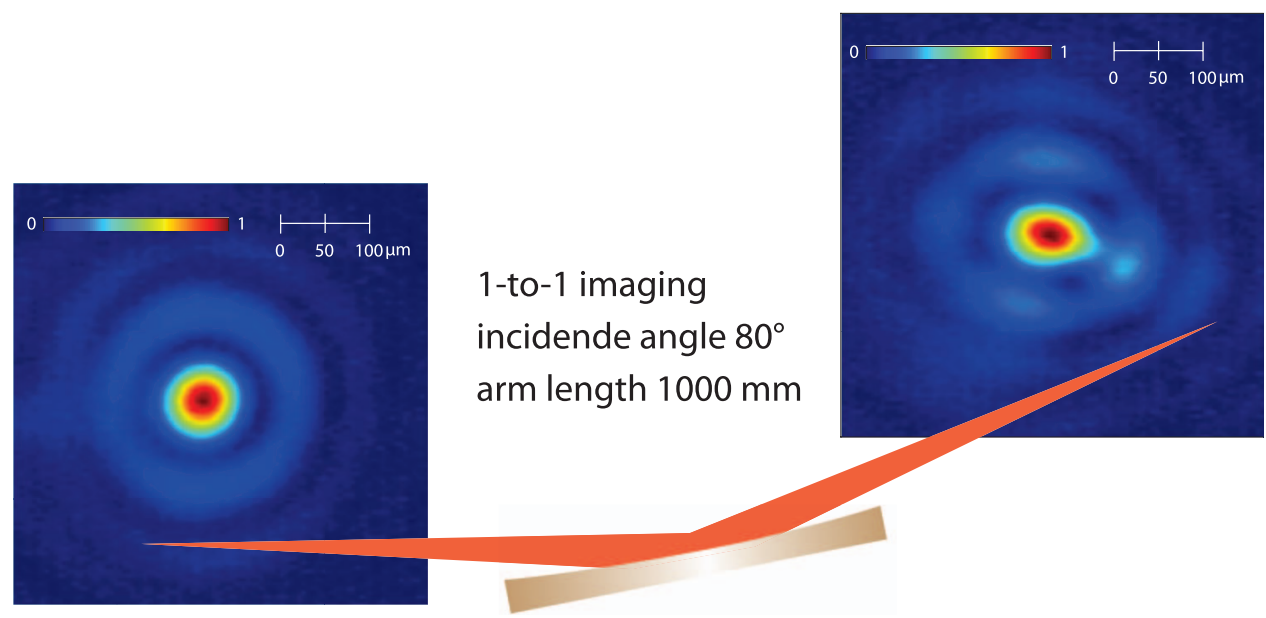

FIG. 9. Beam profile in the 1st (left) and 2nd (right) focus for the delayed beam. For the collinear recombination of XUV and IR beam, the central part of the IR beam is cut out by the DM in Fig. 2 and the beam is clipped by an iris to reduce the intensity. This explains the weak interference rings. As the XUV pulse only overlaps with the central part of the pulse, these pedestals in the transverse beam profile are not important.

decreasing with the IR field strength. This proves that the temporal structure of the XUV pulse as well as of the IR pulse is not significantly distorted.

It should be pointed out that the difference in the intensity distributions of the photoelectron spectra from the two targets is not due to the reflectivity characteristics of the second toroidal mirror, but rather resulting from the different transmission efficiencies of the two electron analyzers. The hemispherical analyzer used in the second interaction region is optimized for solid samples. In order to operate the analyzer with a low-density gas target and obtain a suitable count rate, one has to apply additional acceleration voltages. These change the transfer function of the spectrometer and thus the relative strength of the photoelectron bands. However, for extracting the desired phase information from RABBITT traces the relative strength of the sidebands is not relevant and therefore does not need to be calibrated..$^{9,38}$

The good quality of simultaneously recorded RABBITT traces for the two interaction regions confirms the quality of the reimaging. Note that the cutting-out of the central part of the probe beam for collinear recombination of the XUV and IR beam with the drilled mirror (DM) in Fig. 2 does not adversely affect the beam profile in the focus (Fig. 9). Scans like the presented one were recorded over several hours and no instabilities originating from the beamline or end station were observed. Indeed, the stability of our setup is limited only by the temporal stability of the laser system.

\section{SUMMARY}

We presented a versatile attosecond beamline-referred to as the attoline-suitable for time-resolved measurements on a variety of systems and employing different techniques such as photoelectron/photoion spectroscopy and transient absorption spectroscopy. In particular, our attoline contains two independent interaction regions where the second one is available for any possible end station. Thoughtful design of the interface between beamline and end station allows us to conduct simultaneous photoemission experiments on two different physical systems and opens new perspectives in attosecond spectroscopy. Indeed, the data reported in this work show that well-understood gas phase measurements can be used for calibration or, alternatively, as a reference. Thus, the relative photoemission delays between two different targets, possibly even in different states, can be measured.

Moreover, we show great flexibility in tailoring the characteristics of our source such as the polarization, the intensity, and the temporal structure by control of the number of attosecond pulses within the APT and SAP generation. The beams are reliably delivered into both foci. During the design of our attoline strong emphasis was put on ease of use. Switching between different measurement techniques is straightforward and quick.

The intended purpose of our beamline lies mainly in pump-probe measurements as shown in Secs. III A and III B. Nevertheless, studying the HHG process itself or conducting high-harmonic spectroscopy ${ }^{44-46}$ is equally possible with our setup. In this sense, our beamline efficiently combines established attosecond spectroscopy techniques while retaining the flexibility to adapt to new techniques. We are currently working on an optical parametric amplification (OPA) setup working in the mid-infrared to add to the flexibility of our source and extend the accessible photon energy range.

In conclusion, we believe that our beamline, especially the ability to perform measurements in two independent targets simultaneously, is a powerful toolset to open attoscience to new scientific communities.

\section{ACKNOWLEDGMENTS}

This research was supported by the NCCR MUST, funded by the Swiss National Science Foundation. M.L. acknowledges support from the ETH Zurich Postdoctoral Fellowship Program.

\footnotetext{
${ }^{1}$ A. McPherson, G. Gibson, H. Jara, U. Johann, T. S. Luk, I. A. McIntyre, K. Boyer, and C. K. Rhodes, J. Opt. Soc. Am. B 4, 595 (1987).
} 
${ }^{2}$ M. Ferray, A. L'Huillier, X. F. Li, L. A. Lompre, G. Mainfray, and C. Manus, J. Phys. B: At., Mol. Opt. Phys. 21, L31 (1988).

${ }^{3}$ L. Gallmann, C. Cirelli, and U. Keller, Annu. Rev. Phys. Chem. 63, 447 (2012).

${ }^{4}$ F. Calegari, F. Ferrari, M. Lucchini, M. Negro, C. Vozzi, S. Stagira, G. Sansone, and M. Nisoli, "Principles and Applications of Attosecond Technology" in Advances in Atomic, Molecular, and Optical Physics, edited by E. Arimondo, P. R. Berman, and C. C. Lin (Academic Press, San Diego, 2011), Vol. 60, pp. 371-413.

${ }^{5}$ F. Krausz and M. Ivanov, Rev. Mod. Phys. 81, 163 (2009).

${ }^{6}$ P. B. Corkum and F. Krausz, Nat. Phys. 3, 381 (2007).

${ }^{7}$ A. H. Zewail, J. Phys. Chem. A 104, 5660 (2000).

${ }^{8}$ P. Antoine, A. L'Huillier, and M. Lewenstein, Phys. Rev. Lett. 77, 1234 (1996).

${ }^{9}$ P. Paul, E. Toma, P. Breger, G. Mullot, F. Augé, P. Balcou, H. Muller, and P. Agostini, Science 292, 1689 (2001).

${ }^{10}$ F. Calegari, M. Lucchini, M. Negro, C. Vozzi, L. Poletto, O. Svelto, S. D. Silvestri, G. Sansone, S. Stagira, and M. Nisoli, J. Phys. B: At., Mol. Opt. Phys. 45, 074002 (2012)

${ }^{11}$ T. Shimizu, T. Okino, K. Furusawa, H. Hasegawa, Y. Nabekawa, K. Yamanouchi, and K. Midorikawa, Phys. Rev. A 75, 033817 (2007).

${ }^{12}$ P. Tzallas, E. Skantzakis, L. A. A. Nikolopoulos, G. D. Tsakiris, and D. Charalambidis, Nat. Phys. 7, 781 (2011)

${ }^{13}$ E. J. Takahashi, P. Lan, O. D. Mücke, Y. Nabekawa, and K. Midorikawa, Nat. Commun. 4, 2691 (2013).

${ }^{14}$ J. Itatani, F. Quéré, G. L. Yudin, M. Y. Ivanov, F. Krausz, and P. B. Corkum, Phys. Rev. Lett. 88, 173903 (2002).

${ }^{15}$ L. Gallmann, J. Herrmann, R. Locher, M. Sabbar, A. Ludwig, M. Lucchini, and U. Keller, Mol. Phys. 111, 2243 (2013).

${ }^{16}$ P. Eckle, A. N. Pfeiffer, C. Cirelli, A. Staudte, R. Drner, H. G. Muller, M. Bttiker, and U. Keller, Science 322, 1525 (2008).

${ }^{17}$ A. N. Pfeiffer, C. Cirelli, M. Smolarski, D. Dimitrovski, M. Abu-samha, L. B. Madsen, and U. Keller, Nat. Phys. 8, 76 (2012)

${ }^{18}$ A. Landsman, M. Weger, J. Maurer, R. Boge, A. Ludwig, S. Heuser, C. Cirelli, L. Gallmann, and U. Keller, preprint arXiv:1301.2766 [physics.atom-ph] (2013).

${ }^{19}$ A. N. Pfeiffer, C. Cirelli, M. Smolarski, R. Dorner, and U. Keller, Nat. Phys. 7, 428 (2011).

${ }^{20}$ K. Klünder, J. M. Dahlström, M. Gisselbrecht, T. Fordell, M. Swoboda, D. Guénot, P. Johnsson, J. Caillat, J. Mauritsson, A. Maquet, R. Taïeb, and A. L'Huillier, Phys. Rev. Lett. 106, 143002 (2011).

${ }^{21}$ Y. Mairesse and F. Quéré, Phys. Rev. A 71, 011401 (2005).

${ }^{22}$ G. Sansone, F. Kelkensberg, J. Pérez-Torres, F. Morales, M. Kling, W. Siu, O. Ghafur, P. Johnsson, M. Swoboda, E. Benedetti, F. Ferrari, F. Lépine, J. Sanz-Vicario, S. Zherebtsov, I. Znakovskaya, A. L'Huillier, M. Ivanov, M. Nisoli, F. Martín, and M. Vrakking, Nature (London) 465, 763 (2010).

${ }^{23}$ A. Cavalieri, N. Muller, T. Uphues, V. Yakovlev, A. Baltuska, B. Horvath, B. Schmidt, L. Blumel, R. Holzwarth, S. Hendel, M. Drescher, U. Kleineberg, P. Echenique, R. Kienberger, F. Krausz, and U. Heinzmann, Nature (London) 449, 1029 (2007).

${ }^{24}$ M. Fieß, M. Schultze, E. Goulielmakis, B. Dennhardt, J. Gagnon, M. Hofstetter, R. Kienberger, and F. Krausz, Rev. Sci. Instrum. 81, 093103 (2010).
${ }^{25}$ E. Magerl, S. Neppl, A. L. Cavalieri, E. M. Bothschafter, M. Stanislawski, T. Uphues, M. Hofstetter, U. Kleineberg, J. V. Barth, D. Menzel, F. Krausz, R. Ernstorfer, R. Kienberger, and P. Feulner, Rev. Sci. Instrum. 82, 063104 (2011).

${ }^{26}$ M. Hentschel, R. Kienberger, C. Spielmann, G. Reider, N. Milosevic, T. Brabec, P. Corkum, U. Heinzmann, M. Drescher, and F. Krausz, Nature (London) 414, 509 (2001).

${ }^{27}$ F. Frank, C. Arrell, T. Witting, W. A. Okell, J. McKenna, J. S. Robinson, C. A. Haworth, D. Austin, H. Teng, I. A. Walmsley, J. P. Marangos, and J. W. G. Tisch, Rev. Sci. Instrum. 83, 071101 (2012).

${ }^{28}$ I. J. Sola, E. Mevel, L. Elouga, E. Constant, V. Strelkov, L. Poletto, P. Villoresi, E. Benedetti, J.-P. Caumes, S. Stagira, C. Vozzi, G. Sansone, and M. Nisoli, Nat. Phys. 2, 319 (2006).

${ }^{29}$ H. Telle, G. Steinmeyer, A. Dunlop, J. Stenger, D. Sutter, and U. Keller, Appl. Phys. B: Lasers Opt. 69, 327 (1999).

${ }^{30}$ C. Hauri, W. Kornelis, F. Helbing, A. Heinrich, A. Couairon, A. Mysyrowicz, J. Biegert, and U. Keller, Appl. Phys. B 79, 673 (2004).

${ }^{31}$ C. Hauri, A. Guandalini, P. Eckle, W. Kornelis, J. Biegert, and U. Keller, Opt. Express 13, 7541 (2005).

${ }^{32}$ M. Nisoli, S. De Silvestri, and O. Svelto, Appl. Phys. Lett. 68, 2793 (1996).

${ }^{33}$ G. Sansone, E. Benedetti, F. Calegari, C. Vozzi, L. Avaldi, R. Flammini, L. Poletto, P. Villoresi, C. Altucci, R. Velotta, S. Stagira, S. De Silvestri, and M. Nisoli, Science 314, 443 (2006).

${ }^{34}$ E. Goulielmakis, Z. Loh, A. Wirth, R. Santra, N. Rohringer, V. Yakovlev, S. Zherebtsov, T. Pfeifer, A. Azzeer, M. Kling, S. Leone, and F. Krausz, Nature (London) 466, 739 (2010).

${ }^{35}$ M. Lucchini, J. Herrmann, A. Ludwig, R. Locher, M. Sabbar, L. Gallmann, and U. Keller, New J. Phys. 15, 103010 (2013).

${ }^{36}$ J. Herrmann, M. Weger, R. Locher, M. Sabbar, P. Rivière, U. Saalmann, J.-M. Rost, L. Gallmann, and U. Keller, Phys. Rev. A 88, 043843 (2013)

${ }^{37}$ M. Holler, F. Schapper, L. Gallmann, and U. Keller, Phys. Rev. Lett. 106, 123601 (2011).

${ }^{38}$ H. Muller, Appl. Phys. B 74, s17 (2002).

${ }^{39}$ O. Tcherbakoff, E. Mével, D. Descamps, J. Plumridge, and E. Constant, Phys. Rev. A 68, 043804 (2003).

${ }^{40}$ V. Strelkov, A. Zaïr, O. Tcherbakoff, R. López-Martens, E. Cormier, E. Mével, and E. Constant, J. Phys. B: At., Mol. Opt. Phys. 38, L161 (2005).

${ }^{41}$ D. Kane, IEEE J. Quantum Electron. 35, 421 (1999).

${ }^{42}$ K. DeLong, D. Fittinghoff, and R. Trebino, IEEE J. Quantum Electron. 32, 1253 (1996).

${ }^{43}$ R. López-Martens, K. Varjú, P. Johnsson, J. Mauritsson, Y. Mairesse, P. Salières, M. B. Gaarde, K. J. Schafer, A. Persson, S. Svanberg, C.-G. Wahlström, and A. L'Huillier, Phys. Rev. Lett. 94, 033001 (2005).

${ }^{44}$ J. Itatani, J. Levesque, D. Zeidler, H. Niikura, H. Pepin, J. C. Kieffer, P. B. Corkum, and D. M. Villeneuve, Nature (London) 432, 867 (2004).

${ }^{45} \mathrm{~S}$. Haessler, J. Caillat, W. Boutu, C. Giovanetti-Teixeira, T. Ruchon, T. Auguste, Z. Diveki, P. Breger, A. Maquet, B. Carré, R. Taïeb, and P. Salières, Nat. Phys. 6, 200 (2010).

${ }^{46}$ C. Vozzi, M. Negro, F. Calegari, G. Sansone, M. Nisoli, S. De Silvestri, and S. Stagira, Nat. Phys. 7, 822 (2011). 